\title{
In-beam tests of a PET demonstrator (LAPD) for hadrontherapy beam ballistic control: data comparison to Geant4 Monte-Carlo predictions.
}

\author{
Sébastien Binet, Arthur Bongrand, Emmanuel Busato, Christophe Insa, \\ Daniel Lambert, Magali Magne, Franck Martin, Gérard Montarou, Arnaud Rozes \\ Université Clermont Auvergne \\ Laboratoire de Physique de Clermont-Ferrand \\ Clermont-Ferrand, France \\ franck.martin@clermont.in2p3.fr
}

\begin{abstract}
This paper describes a small prototype of an in beam PET like detector, named "Large Acceptance Pixelized Detector" (LAPD), developed to test technical concepts for the ion range control in the context of cancer treatments using proton or ion beams. The mechanical characteristics of this detector together with the read-out electronics are first presented. Then, results of a first experiment, performed on a $65 \mathrm{MeV}$ proton beamline, are reported. Finally, we discuss the ability of Geant4 Monte-Carlo to reproduce the experimental data.
\end{abstract}

Index Terms-In-beam PET, hadrontherapy, Geant4, simulation.

\section{INTRODUCTION}

Ion beam therapy is a non-invasive cancer treatment used for the treatment of unresectable and radioresistant tumors, or for pediatric cancer treatments. It minimizes damages to healthy tissues, but the uncertainty on the ion range, of the order of $3 \%$ of this range, requires a very good quality control of the treatment. Positron Emission Tomography (PET) is a medical imaging modality which has already been applied for ion range verification in the context of ion beam therapy treatments. This modality has been chosen as there is a direct correlation between the dose distribution in the biological tissues and the spatial distribution of the $\beta^{+}$emitting nuclei induced by the nuclear reactions between particles of the beam and nuclei of the target [1].

Different approaches of PET modalities have been considered up to now for ballistic control, usually referred as in-beam PET [2]-[7] and off-beam PET. The later could be separated in two categories: either the PET scan is performed directly in the treatment room (in-room PET) or off the treatment room using standard clinical PET (off-room PET).

The in-beam PET, which means that the PET acquisition is done during the treatment, simultaneously to the irradiation, is the only solution from the point of view of in-situ (and eventually and ideally real time) beam ballistic control, but

This work was supported by the LABEX PRIMES (ANR-11-LABX-0063) of Université de Lyon, within the program "Investissements d'Avenir" (ANR11-IDEX-0007) operated by the French National Research Agency (ANR), and by an emergent project grant of the University of Clermont-Auvergne. it suffers for a large background from beam particle induced nuclear reactions, as the beam induces in the tissues not only $\beta^{+}$emitters, but also a large amount of secondary particles as protons, neutrons, and gamma from deexcitation of excited nuclear levels (known as "prompt" $\gamma$ as they are almost in time with the beam spill). The off-beam PET does not suffer from this beam-induced background, but there is a delay between the treatment and the PET scan, which induces $\beta^{+}$ activity wash-out, degrading the available statistics and the endpoint ballistic information. Moreover, it is no more an in-situ beam ballistic control. In the in-room PET solution, the statistics has been shown to be sufficient and the induced activity is moderately washed-out, but the patients need to stay longer in the treatment room and it is still not a real time beam ballistic control.

Recently, many small acceptance dedicated system have been developed and used in the context of in-beam ballistic control [8]-[11]. In order to test new trigger strategies for inbeam and in-time measurements and following the work of [8], [9], a crystal-PMT based demonstrator was built. The aim of this detector is to study the potentiality of a new sampling electronics and its associated trigger to extract true $511 \mathrm{keV} \gamma$ coincidences from the nuclear induced background during inbeam PET acquisition. This detector should also be efficient whatever the beam structure is.

The paper is organized as follows. Section II presents the detector mechanics, its electronics and two versions of the data acquisition system. Section III shows some results obtained with a $65 \mathrm{MeV}$ proton beamline at the IMPT Nice, France. Last section discusses simulation results .

\section{DETECTOR DESCRIPTION}

\section{A. mechanical assembly}

The demonstrator has been designed with a robust and low cost detection technology to be easily extended on a larger scale. The LAPD is made of 240 identical channels. Each channel is constituted of a $13 \times 13 \times 15 \mathrm{~mm}^{3}$ LYSO crystal 

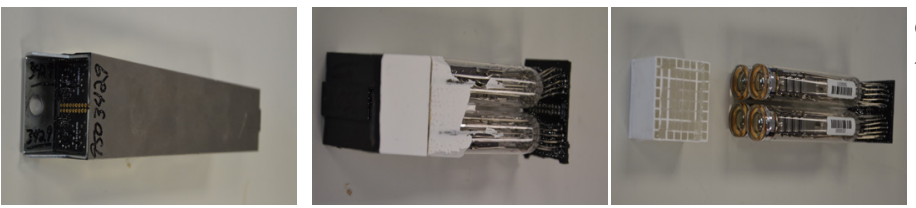

Fig. 1. Pictures of the reconditionning steps of the PMTs of the Siemens $\mathrm{HR}^{+}$PET camera. Left: original block. Middle: original block made of a BGO arrays coupled in Anger mode to 4 PMTs, embedded in silicon. Right: BGO crystal and cleaned PMT quartet.

coupled to a photomultiplier tube (PMT). The PMTs (Hamamatsu R1450) originate from a reformed HR+ scanner from Siemens. All the PMTs have been separated from their BGO crystal block (Fig. 1), before being tested on two different test benches, on which the PMTs are illuminated with $465 \mathrm{~nm}$ blue LED. First test bench was dedicated to the measurement of the gain as a function of the applied high voltage, and the second to the control of the PMT gain drift. Then, the PMTs have been assembled on a new board to form a new "quartet". The four PMTs of the same quartet have been chosen with similar gains, as they share the same input high voltage (HV) line.

Each PMT of the quartet is then coupled to its LYSO crystal, the crystal being glued with ARALDITE®2000 20281 optical glue (Fig. 2). Three solutions have been tested for the crytal wrapping, with respect to the light yield: Teflon®, white silicon and $\mathrm{TiO}_{2}$ white paint. Paint and Teflon ${ }^{\circledR}$ gave the better results, and Teflon ${ }^{\circledR}$ wrapping has been chosen. The crystal is covered by three $0.2 \mathrm{~mm}$ thickness Teflon ${ }^{\circledR}$ layers. Each quartet is finally connected to its front-end electronics board, as shown on figure 3 .
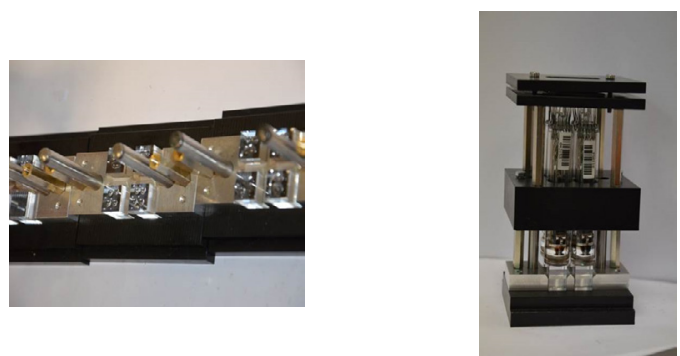

Fig. 2. Left: picture of the LYSO crystals, positionned in the mounting tool. Right: PMT in position in the mounting tool

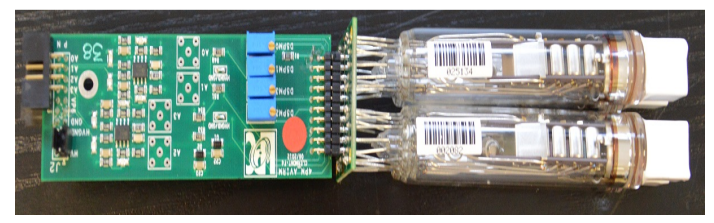

Fig. 3. Picture of one quartet connected to its front-end (HV divider and differential amplifier) board.

Five quartets are then assembled to form a $188.5 \mathrm{~mm}$ long raw, as shown on figure 4 . The complete detector is constituted of two half-rings of 6 raws each, forming the two heads of the system.

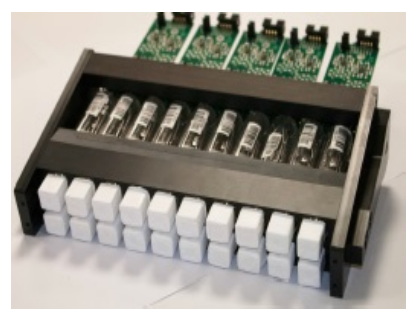

Fig. 4. Picture of one raw of 5 quartets.

Schematic view of the crystals of one half-ring is shown on figure 5. On this figure, the beam comes from the right, and defines the $z$ axis. To improve the spatial sampling, each raw is shifted by $\pm 6.5 \mathrm{~mm}$ in the $z$ direction with respect to the middle raw of each $1 / 4$ of the detector. In the transverse $(x$, $y$ ) plane with respect to the beam axis, the middle of each quartet is located at a radius of $148.4 \mathrm{~mm}$ from the $z$ axis.

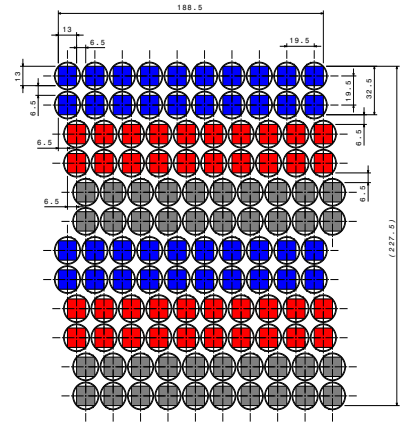

Fig. 5. View of the crystal of the right side of the LAPD, if the hadron beam is coming from the right. The red layer is taken as the reference in the $z$ direction, The blue layer is sifted by $+6.5 \mathrm{~mm}$ toward the left, and the grey layer is shifted by $-6.5 \mathrm{~mm}$ toward the right.

The two half-rings are then supported by the mechanical struture shown on figure 6 , which also supports all the cables. The inner diameter of the detector is slightly less than 300 $\mathrm{mm}$, and the height and width are about 1 meter. A movable target support is also included, and the total weight, including the cables, is about $80 \mathrm{~kg}$. The whole detector is on a movable table that allows adjusting the position on a beamline.

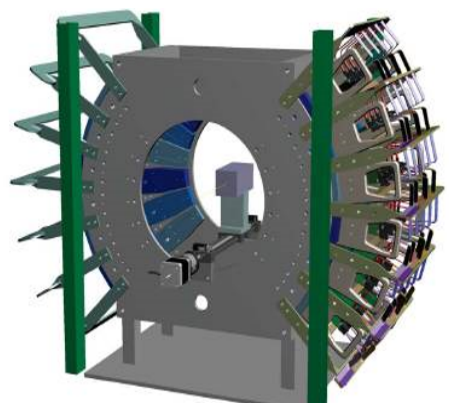

Fig. 6. CAD view of the complete LAPD 


\section{B. Detector electronics}

The high voltage is regulated by a custom made high voltage board, which provides 15 output lines for one HV input. Four boards are needed for the complete LAPD.

The PMT signal is sent to an Analog Sampling Module shown on figure 7 , specially designed in our laboratory for this application. This VME $6 \mathrm{U}$ board receives up to 24 differential analog input signals. This board has two main functionalities: the first is to sample in parallel and at high rate the 24 input analog signals and the second is to perform a first energy based selection of the events, for triggering purpose. This board is built around a FPGA Cyclone 4 of Altera. The ASM board is composed of a mother board with the FPGA and all the threshold electronics, and three mezzanines for the sampling and digitazing electronics. The mezzanines received 8 input channels connected to a DRS4 chip [12]. This chip is a switched capacitor array used to continuously store the analog signal until a trigger decision is taken. The samples are digitized by a 12 bits - $30 \mathrm{MHz}$ ADC. The sampling rate is adjustable between 1 and $5 \mathrm{GHz}$, but is fixed to $5 \mathrm{GHz}$ in our application, and the maximum buffer size is 1024 samples.

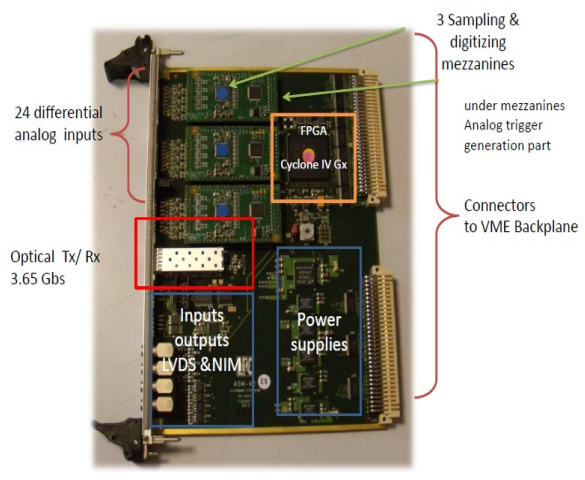

Fig. 7. Picture of the ASM board.

The choice has been made to use 12 ASM boards for the complete LAPD, in such a way that all the quartets of the same raw (20 channels) are connected to an unique ASM board. The 12 ASM boards are housed in a VME crate.

\section{Trigger and data acquisition system}

The trigger signal generation is an important part of the ASM characteristics.

To generate this trigger signal each analog input signal is duplicated at the ASM entrance. The first duplicated signal goes to the DRS4 and the second is sent to 2 comparators, whose thresholds are adjusted through a 12 bits programmable DAC controlled via the FPGA. Signal with amplitude between the low and high levels are accepted.

The ASM board then performs the logical OR of the 20 comparator outputs, and can generate a "single channel" trigger. This logical signal is then sent to another FPGA located on a trigger board (the THOR board, which also provides the clock to synchronize the ASM boards). The THOR board implements the logical OR of the 10 ASM of one half-ring, and the AND between the two half-rings to detect eventual coincidences. The trigger scheme is completed with a geometrical selection. Three selection patterns are implemented : if a crystal is touched on one half-ring and a second crystal is impacted in coincidence on the other halfring, a trigger signal is finally generated:

- whatever the position of the second crystal is (named 1 versus all: 1LvA);

- if the second crystal is located in the diametrally opposited raw of quartets, or in the two neighboring raws (named 1 versus 3, 1Lv3);

- if the second crystal is located in the diametrally opposited raw of quartets only (named 1 versus 1, 1Lv1).

In what follows, only the second selection pattern is used, as it restricts the field of view roughtly to the PMMA target. When an event is triggered, the data are sent to the VME CPU board, and then to the acquisition PC using TCP/IP protocol. In this first VME-based DAQ configuration, the sampled data are read back by the VME bus in BLT32 mode, which induces a the large dead time. This is a severe limitation, as the acquisition rate is limited to $25-30 \mathrm{~Hz}$. The results shown in the next section are obtained with this low-bandwidth DAQ. The complete description of the detector construction and VMEbased acquisition system can be found in [13].

A second DAQ system, based on $\mu$ TCA technology, is under development. The first layer is still the ASM board, but it uses the $3 \mathrm{~Gb}^{-1}{ }^{-1}$ optical ouput of the ASM board rather than the VME back pannel, to send the data to an AMC40 board (rather than the VME CPU board) which then send them to the acquisition PC trough a $10 \mathrm{~Gb}^{-1}{ }^{-1}$ optical link, using UDP connectionless protocol (rather than TCP/TP protocol). The other change is that only the data corresponding to the two triggering channels are sent, rather that all the data corresponding to the channels above an operator defined threshold, as it is done presently. Such a system has been tested in November 2018, with a gain in the data acquisition frequency of a factor one hundred.

\section{EXPERIMENT AT THE IMPT With a 65 MEV PROTON BEAMLINE}

\section{A. Detector installation}

In March 2017, the LAPD has been installed on the Medicyc beamline at the IMPT ("Institut Méditerranéen de ProtonThérapie"', a cancer treatment center in Nice, France), as shown on figure 8 , and first beam tests of the full LAPD system were performed in March 2017 and May 2017. This beamline provides a $65 \mathrm{MeV}$ proton beam for ocular tumor treatments, with a 4 ns spill every $40 \mathrm{~ns}$. The beam spot has a transverse extension, measured with gafchromic films, of $2.5 \times 3.8 \mathrm{~cm}^{2}$ full width half maximum at the entrance of the detector. A reference time is provided by the accelerator radio frequency signal, which is registered on one of the empty channels of one ASM board. For the experiments presented 
in this paper, a beam intensity of about $5 \mathrm{nA}$ was used. This corresponds to a number of protons per spill of approximately 1250 .

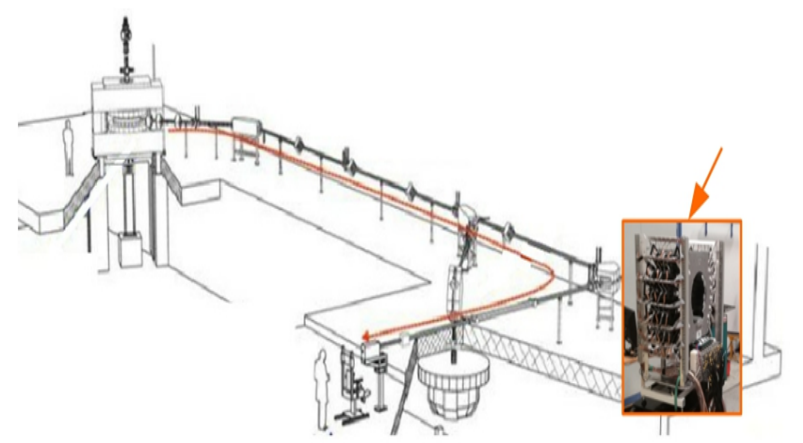

Fig. 8. The LAPD is installed on the Medicyc beamline, just before the treatment room. The last dipole magnet on the beamline is swithed off when the daily treatments are finished, to deliver the beam to the experiment rather than in the treatment room.

\section{B. Event selection}

At the trigger level, the coincidence window is set to 20 ns, and the geometrical trigger equation if the 1Lv3 selection pattern described in II-C.

Offline analysis is then performed, requiering an energy deposition in each cristal of $511 \mathrm{keV} \pm 90 \mathrm{keV}$, a time difference between the two detected photons less than $3.6 \mathrm{~ns}$ and a minimum approach distance between the reconstructed LOR and the beam axis less than $1.5 \mathrm{~cm}$. Also, each photon arrival time should be between 20 and 50 ns after the reference time. Finally, only events with photons registered during the beam pause (see fig. 9) and one valid LOR are kept. Once the LOR are selected, the $z$ profile of the activity distribution is reconstructed by a Kernel estimation of the underlying probability density function [14].

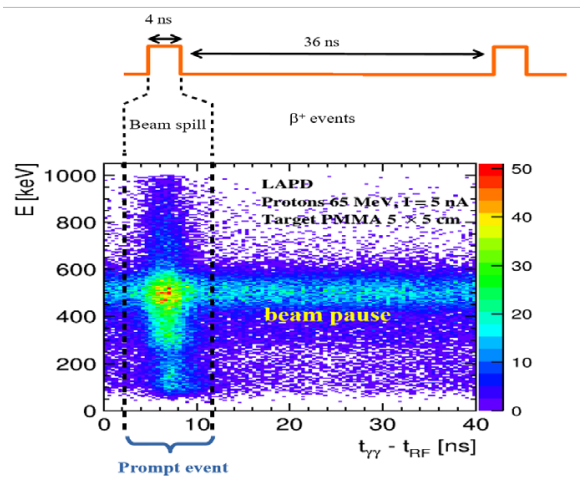

Fig. 9. Energy deposition in the two cristals, as a function of the difference between the mean photon arrival time and the reference time. This reference time is given by the accelerator RF. The events between the two black dashed lines are rejected, as they likely correspond to prompt gamma events (the beam spill is $4 \mathrm{~ns}$ long, and the beam pause is $36 \mathrm{~ns}$ long).

\section{Results}

We describe here one of the experiments performed at the IMPT Nice in 2017. PMMA targets of $5 \times 5 \times 5 \mathrm{~cm}^{3}$ were displaced along the beam axis, and the activity distributions were reconstructed for each of the 10 different target positions. When shifting the target by a certain distance, the Bragg peak is expected to shift by the same distance as the energy lost by $65 \mathrm{MeV}$ protons in air in distances of the order of a few centimeters is negligible compared to their initial energy. The $\beta^{+}$activity profile is also expected to shift by exactly the same distance. The ability of the LAPD to reconstruct the $\beta^{+}$ activity profile and thus its ability to control the proton range can therefore be evaluated by comparing the known target shifts to the reconstructed ones.

The figure 10 shows the 10 reconstructed activity profiles. The position of the distal fall-off at half maximum is calculated, and is taken as an estimator of the target position. The figure 11 shows the positions measured with the reconstructed activity p.d.f as a function of the true position of the target. A nice agreement, with a precision of $1 \mathrm{~mm}$ at the field of view center $( \pm 2 \mathrm{~cm}$ around the detector center), and $2 \mathrm{~mm}$ at most otherwise, is obtained.

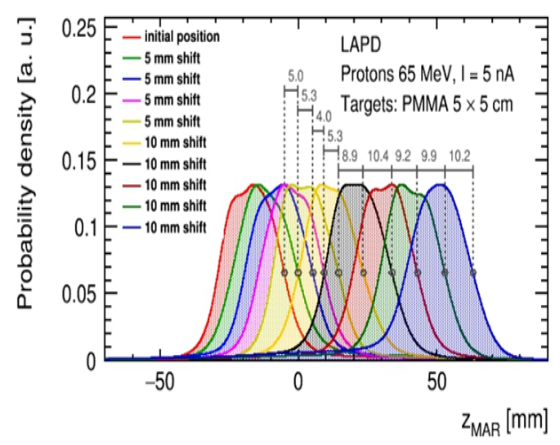

Fig. 10. PMMA targets of $5 \times 5 \times 5 \mathrm{~cm}^{3}$ were displaced along the beam axis, and the activity p.d.f was reconstructed by a Kernel estimation for each of the 10 different target positions. The position of the distal fall-off of the p.d.f at half maximum is calculated, and is taken as an estimator of the target position. Estimated shifts are indicated on the figure, to be compare to the real target displacement indicated in the legend.

\section{Monte-Carlo simulation}

Geant4 10.4.p02 was used for the Monte-Carlo simulation. Protons of $55 \mathrm{MeV}$, hitting of PMMA target, were first simulated. The goal was to compare the Geant4 predictions to the experimental results obtained by Dendooven et al. [15], reported in the table I. The relative difference between Geant4 and the experimental results are shown on figure 12, for the Binary Cascade (BIC) and the BIC High Precision (BIC_AllHP) models. Both models show rather large discrepancies for the $\beta^{+}$emitter production rates. The BIC model has been found to predict the short-lived isotope production in the correct order (BIC_AllHP predicts a huge amount of ${ }^{8} \mathrm{~B}$ ) and with a correct order of magnitude. For these reasons, 


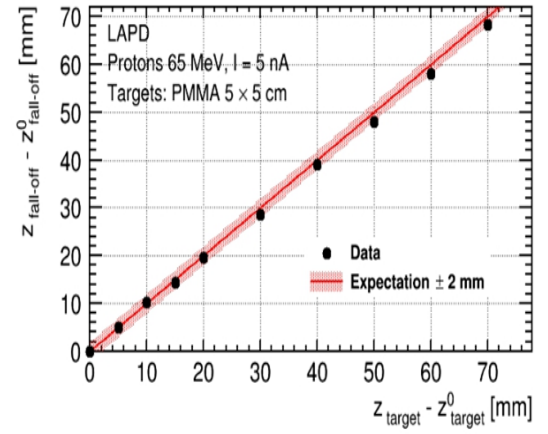

Fig. 11. Estimated displacements, measured with the reconstructed activity p.d.f as a function of the true position of the target. A precision of $1 \mathrm{~mm}$ at the field of view center $( \pm 2 \mathrm{~cm}$ arround the detector center), and $2 \mathrm{~mm}$ otherwise, is obtained.

and also because the simulation with the BIC model is faster than with the BIC_AllHP model, the BIC model was chosen. The different models predict also different shapes for the $\beta^{+}$ activity profiles, as shown on figures 13 and 14. In both cases, it should be noted that the short-lived ${ }^{12} \mathrm{~N}$ profile does not show a sharp fall-off, as for the long-lived ${ }^{11} \mathrm{C}$ or ${ }^{15} \mathrm{O}$ emitters. This is due to the high energy of the emitted $\beta^{+}$.

\begin{tabular}{|c|c|c|c|}
\hline Isotope & $\begin{array}{c}\text { Production rate } \\
\left(\beta^{+} / \text {proton }\right) \text { at } 55 \mathrm{MeV}\end{array}$ & $\mathrm{T}_{1 / 2}$ & $\begin{array}{c}\beta^{+} \text {energy } \\
(\mathrm{MeV})\end{array}$ \\
\hline${ }^{11} \mathrm{C}$ & $4.4110^{-3}$ & $20.33 \mathrm{~min}$ & 0.4 \\
${ }^{15} \mathrm{O}$ & $3.2610^{-3}$ & $122.24 \mathrm{~s}$ & 0.7 \\
${ }^{12} \mathrm{~N}$ & $4.0010^{-4}$ & $11.00 \mathrm{~ms}$ & 6.00 \\
${ }^{10} \mathrm{C}$ & $1.6910^{-4}$ & $19.29 \mathrm{~s}$ & 0.8 \\
${ }^{14} \mathrm{O}$ & $2.7010^{-5}$ & $70.61 \mathrm{~s}$ & 0.7 \\
${ }^{8} \mathrm{~B}$ & $6.3010^{-6}$ & $770 \mathrm{~ms}$ & 4.5 \\
${ }^{17} \mathrm{~F}$ & $<10^{-6}$ & & \\
\hline
\end{tabular}

TABLE I

Production RATES OF THE MAIN $\beta^{+}$EMITTERS PRODUCED IN A PMMA TARGET, MEASURED By By DENDOOVEn et al. [15], ON A $55 \mathrm{MEV}$ PROTON BEAM. THE HALF-LIVES AND THE EMITTED $\beta^{+}$ENERGIES ARE ALSO INDICATED. THE SHORT-LIVED ${ }^{12} \mathrm{~N}$ COULD BE OF HUGE INTEREST FOR in-beam PET MEASUREMENT, BUT HAS A HIGH $\beta^{+}$ENERGY, WHICH MAKES IT DIFFICULT TO USE TO DETERMINE THE BRAGG PEAK POSITION.

The detector, with its time and energy resolutions, the Medicyc beamline, the data acquisition system have been simulated. Simulated events have been submitted to the same selection criteria as for the data, to select the photons from which the LOR are built. The discrete $z$ profile has been reconstructed for different target positions along the beam axis, and the final activity distribution is reconstructed by a Kernel estimation of the underlying probability density function. This p.d.f are shown on the figure 15. The position of the distal falloff of the p.d.f at half maximum is calculated, and is taken as an estimator of the target position. The figure 16 shows the positions measured with the reconstructed activity p.d.f as a function of the true position of the target, for the simulated and real events. A very good agreement between the data and the Monte-Carlo prediction is obtained. The predicted precision is $1 \mathrm{~mm}$ at the field of view center $( \pm 2 \mathrm{~cm}$ around the detector

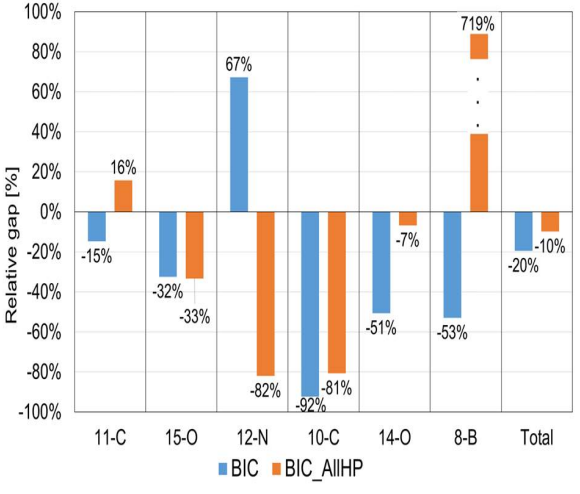

Fig. 12. Relative difference between the Geant 4 predictions and the experimental results, as measured by Dendooven et al., for the production rate $\left(\beta^{+} /\right.$ proton) at $55 \mathrm{MeV}$. Results obtained with the BIC and BIC_AllHP models implemented in Geant4 are shown.

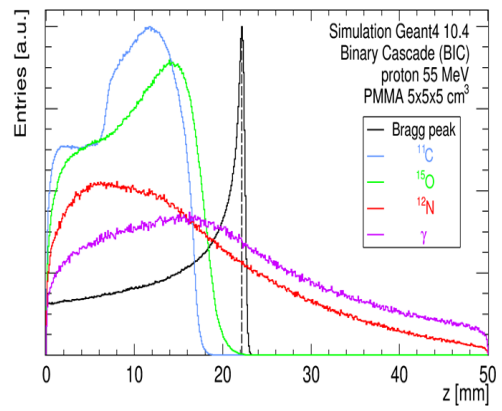

Fig. 13. $\beta^{+}$activity profile obtained with the BIC model in Geant4. The three mains $\beta^{+}$emitters are shown, as well as the prompt $\gamma$, which are the fourth souce of $\beta^{+}$through the pair creation process.

center), and $2 \mathrm{~mm}$ at most otherwise.

\section{CONCLUSIONS}

The construction and first proton beam tests of an in-beam PET demonstrator (LAPD) for hadrontherapy beam ballistic control have been described. The ability of the LAPD system to measure shifts in the Bragg peak position during irradiation has been evaluated with PMMA targets by varying the position of the targets along the beam axis. The shift is measured with a precision of approximately $1 \mathrm{~mm}$ at the center of the field of view. This precision is comparable to the uncertainty currently used in treatment planning for ocular tumors and better than the one used for deeper tumors. The current DAQ system, used for these tests, is limited by the dead time coming from the data transfer via the VME backplane. This prevents performing in-beam PET measurements with timescales appropriate to clinical situations. In order to reduce the dead time, a new DAQ system based on the $\mu$ TCA technology is developed. First performances of this new high bandwidth system have been evaluated on the $65 \mathrm{MeV}$ (Medicyc) proton beamline, and showed an increase in the data acquisition frequency of a factor 100. Geant4 predictions have been compared to $55 \mathrm{MeV}$ 


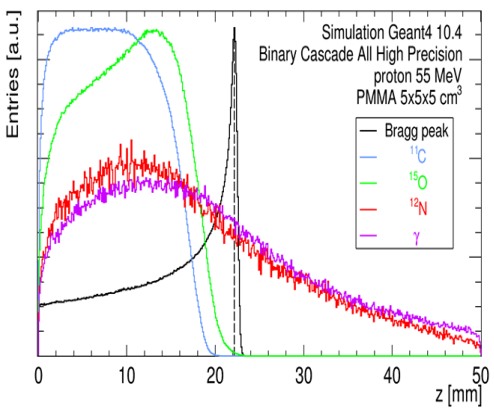

Fig. 14. $\beta^{+}$activity profile obtained with the BIC High Precision model in Geant4. The three mains $\beta^{+}$emitters are shown, as well as the prompt $\gamma$, which are the fourth souce of $\beta^{+}$through the pair creation process.

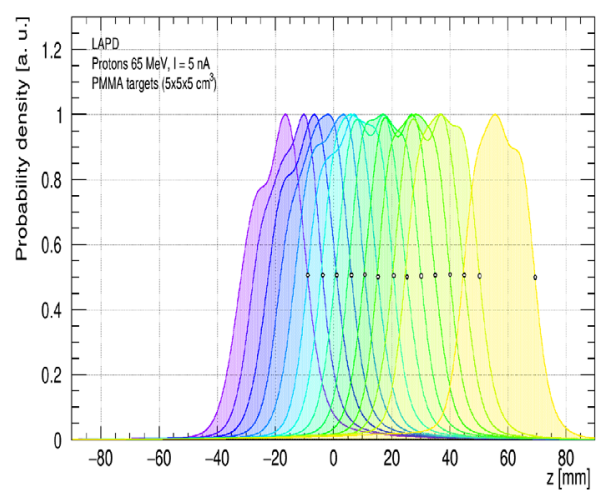

Fig. 15. Reconstructed $\beta^{+}$activity p.d.f for various PMMA target positions along the beam axis, for simulated data. The simulation is performed in the same irradiation conditions as for the data $(5 \mathrm{nA})$.

measurements, showing rather large discrepancies for the $\beta^{+}$ emitter production rates. The BIC model has been found to predict the isotope production in the correct order, and with the correct order of magnitude. This BIC model has been used to simulate the experiment. Data and Monte-Carlo predictions are in good agreement, with again a precision of approximately $1 \mathrm{~mm}$ at the center of the field of view for the Monte-Carlo simulation.

\section{ACKNOWLEDGEMENTS}

We are very gratefull to the whole team of the Centre Antoine Lacassagne at NICE, particularly to R. Trimaud, J. Herault, J.C. Grini, M. Vidal and A. Gérard, for there permanent support.

\section{REFERENCES}

[1] J. Pawelke, W. Enghardt, T. Haberer, B.G. Hasch, R. Hinz, M. Kramer, E. Lauckner, M. Sobiella, In-beam PET imaging for the control of heavyion tumour therapy, IEEE TNS, vol. 44 (4), pp 1492-1498, 1997.

[2] K. Parodi, W. Enghardt and T. Haberer, In-beam PET measurements of $\beta^{+}$radioactivity induced by proton beams, Phys. Med. Biol., vol. 47, pp 21-36, 2002

[3] P. Crespo, G. Shakirin and W. Enghardt, On the detector arrangement for in-beam PET for hadron therapy monitoring, Phys. Med. Biol., vol. 51, pp 2143-2163, 2006.

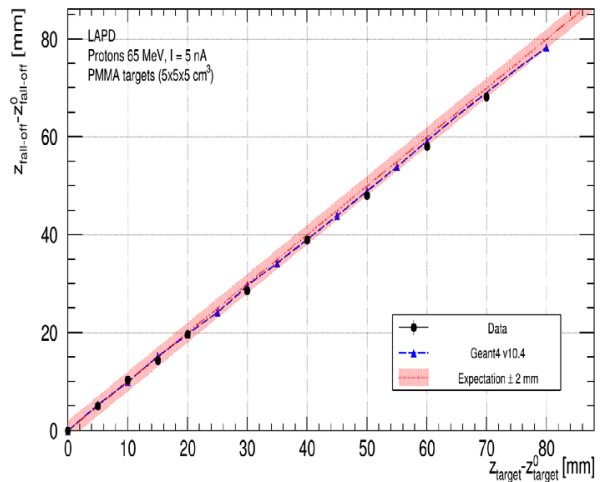

Fig. 16. Estimated displacements, measured with the reconstructed $\beta^{+}$ activity p.d.f as a function of the true position of the target, for simulated events. A precision of $1 \mathrm{~mm}$ at the field of view center $( \pm 2 \mathrm{~cm}$ arround the detector center), and $2 \mathrm{~mm}$ otherwise, is obtained. The agreement between the data and the Monte-Carlo prediction is very good.

[4] P. Crespo, G. Shakirin, F. Fiedler, W. Enghardt and A. Wagner Direct time-of-flight for quantitative, real-time in-beam PET: a concept and feasibility study, Phys. Med. Biol., vol. 52, pp 6795-6811, 2007.

[5] F. Fiedler, G. Shakirin, J. Skowron, H. Braess, P. Crespo, D. Kunath, J. Pawelke, F. Pnisch, W. Enghardt, On the effectiveness of ion range determination from in-beam PET data, Phys. Med. Biol., vol. 55, pp 1989-1998, 2010.

[6] H. Tashima, T. Yamaya, E. Yoshida, S. Kinouchi, M. Watanabe, E. Tanaka, A single-ring OpenPET enabling pet imaging during radiotherapy, Phys. Med. Biol., vol. 57, pp 4705-4718, 2012.

[7] S. Vecchio, F. Attanasi, N. Belcari, M. Camarda, G. A. P. Cirrone, G. Cuttone, F. Di Rosa, N. Lanconelli, S. Moehrs, V. Rosso, G. Russo, A. Del Guerra A PET Prototype for "In-Beam"' Monitoring of Proton Therapy, IEEE TNS, vol. 56 (1), 2009.

[8] L. Lestand, Etude de la mesure en ligne de l'activit $\beta^{+}$induite lors des traitements d'hadronthrapie, en vue de leur contrle balistique., $\mathrm{PhD}$ thesis n 2268, Clermont-Ferrand II University, 2012.

[9] L. Lestand et al., In beam TEP acquisition on 75 A.MeV carbon beam using sampling-Based Read-out Electronics, IEEE Transactions on Radiation and Plasma Medical Sciences, Vol. 1, No. 1, pp. 87-95, 2017.

[10] G. Sportelli, K. Straub, M. Aiello, F. Attanasi, N. Belcari, N. Camarlinghi, G. A. P. Cirrone, G. Cuttone, S. Ferretti, N. Marino, D. Nicolosi, F. Romano, V. Rosso, A. Del Guerra, Full in-beam PET measurements of $62 \mathrm{MeV}$ protons onto a PMMA target, NIM A 718, pp 151-153, 2013.

[11] G. Sportelli, N. Belcari, N. Camarlinghi,G. A. P. Cirrone, G. Cuttone, S. Ferretti, A. Kraan, J.E. Ortuo, F. Romano, A. Santos, K. Straub, A. Tramontana, A. Del Guerra, V. Rosso, First full-beam PET acquisitions in proton therapy with a modular dual-head dedicated system. , Phys. Med. Biol., vol. 59, pp 43-60, 2014.

[12] S. Ritt, Design and performance of the $6 \mathrm{GHz}$ waveform digitizing chip DRS4, Nuclear Science Symposium Conference Record, pp 1512-1515, 2008 and http://www.psi.ch/drs/

[13] G. Montarou et al., Construction and Tests of an In-beam PET-like demonstrator for hadrontherapy beam ballistic control, NIM A 845, pp 673-678, 2017.

[14] E. Busato et al., Construction and First Tests of an PET Demonstrator Dedicated to the Ballistic Control of Hadrontherapy Treatments With $65 \mathrm{MeV}$ Protons, IEEE Transactions on Radiation and Plasma Medical Sciences, Vol. 2, Issue:1, pp 51-60, 2018.

[15] P. Dendooven, H.J. Buitenhuis, F. Diblen, P.N. Heeres, A.K. Biegun, F. Fiedler, M.J. van Goethem, E.R. van der Graaf, S. Brandenburg, Shortlived positron emitters in beam-on PET imaging during proton therapy, Phys. Med. Biol., vol. 60, pp 8923-47, 2015. 